\title{
New Near Open Set In Topological Space
}

\section{Sayed MEL* and Mansour FHAL}

Department Mathematics, College of Science and Arts, Najran University, Saudi Abrabia

\begin{abstract}
The aim of this paper is to introduce new class of near open sets namely, $b^{*}$-open set. And studs same of their properties, also we study the relation between this class among this classes. Also, we introduce some topological properties and we shall study same of their properties.
\end{abstract}

Keywords: $b^{*}$-open set, $b^{*}$-interior, $b^{*}$-closure, $b^{*}$-boundary, $b^{*}$ neighbourhood.

\section{Introduction}

Topological ideas are present not only in almost all areas of today mathematics for example biochemistry [1] information systems [2] and others for more fields of topology applications see ref. [3] and its related links. The subject of topology itself consist of several different branches such as point set topology, algebraic topology and differential topology which have relatively little in common this richness of applications and difference between branches of topology. implied a difficulty to give an accurate definition for topology. In 1937 [4] M.H. Stone introduced the concept of regular open sets. In 1963 [5] Levine introduced the concept of semi open sets. In 1965 [6] Najasted introduced the concept of a-open sets. In 1982 [7,8] Mashhour, Abd El-Monsef and El-Deeb introduced the concept of pre-open sets. In 1983 [9] Abd El-Monsef and et al. introduced the concept of $\beta$-open sets. In 1996 [10] Andrijevic introduced the concept of b-open sets, In 2013 [11] Hariwan Z Ibrahim introduced the concept of Bc-Open Set.

Definition 1.1: A subset $A$ of topological space $(X, \tau)$ is

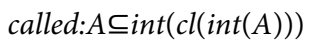

(1) $\alpha$-open if $A \subseteq \operatorname{int}(\operatorname{cl}(\operatorname{int}(A)))$ [6]

(2) preopen if $A \subseteq \operatorname{int}(c l(A))$ [8]

(3) semi open if $A \subseteq \operatorname{cl}(\operatorname{int}(A))$ [5]

(4) Regular open if $A=\operatorname{int}(\operatorname{cl}(A)) \beta[4]$

(5) $\beta$-open (or semi pre open) if, $A \subseteq(\operatorname{cl}(\operatorname{int}(\operatorname{cl}(A)))[9-15]$

(6) $b$-open. $A \subseteq \operatorname{cl}(\operatorname{int}(A))$ Ç $\operatorname{int}(\operatorname{cl}(A))$ [10]

(7) $A$ subset $A$ of a space $X$ is called Bc-open if for each $x \in A$ $\in b O(X)$, there exists a closed set $F$ such that $x \in F \subset A$ [11]

Remark 1.1: The complement of a $\alpha$-open (resp. preopen, semi open, Regular open, $\beta$-open and b-open) sets is called $\alpha$-closed (resp. pre closed, semi closed, Regular closed, $\beta$-closed and b-closed ) sets. The intersection of all $\alpha$-closed (resp. pre closed, semi closed, Regular closed, $\beta$-closed and $b$-closed) sets containing $A$ is called the $\alpha$-closure (resp. pre-closure, semi-closure, Regular closure, $\beta$-closure and b-closure) of $A$ and is denoted by $\alpha c l(A)$ (resp. pcl (A), scl(A), $\operatorname{Rcl}(A), \beta c l(A)$ or spcl $(A)$, and $b c l(A))$.

The union of all a $\alpha$-open (resp. preopen, semi open, Regular open, $\beta$-open, and $b$-open ) sets contained in $A$ is called $\alpha$-intrior (resp. preintrior, semi-intrior, Regular intrior, $\beta$-intrior and $b$-intrior ) of $A$ and is denoted by $\alpha \operatorname{int}(A)($ resp. pint (A), sint (A), $\operatorname{Rint}(\mathrm{A}), \operatorname{\beta int}(A)$ or spint (A), and bint (A)). The family of all a $\alpha$-open (resp. $\alpha$-closed, preopen, pre closed, semi open, semi closed, Regular open, Regular closed, $\beta$-open, $\beta$-closed and $b$-open, $b$-closed $)$ sets is denoted by $\alpha O(X)$ $(\operatorname{resp} \alpha C(X), \mathrm{PO}(\mathrm{X}), \mathrm{PC}(\mathrm{X}), \mathrm{SO}(\mathrm{X}), \mathrm{SC}(\mathrm{X}), \mathrm{RO}(\mathrm{A}), \mathrm{C}(\mathrm{A}), \beta O(A), \beta C(A)$, $\mathrm{bO}(\mathrm{A})$ and $\mathrm{bC}(\mathrm{A}))$. hold:

Proposition 1.1: For sub set $A, B$ a spase $(X, \tau)$, the following statmant

(1) $\operatorname{pcl}(A)=A \cup \operatorname{cl}(\operatorname{int}(A)), \operatorname{pint}(A)=A \cap \operatorname{int}(\operatorname{cl}(A))$ [10].

(2) $\operatorname{spcl}(A)=A \cup \operatorname{int}(\operatorname{cl}(\operatorname{int}(A))), \operatorname{spint}(A)=A \cap \operatorname{cl}(\operatorname{int}(\operatorname{cl}(A)))[10]$.

(3) $\operatorname{pcl}(A \cup B) \subseteq \operatorname{pcl}(A) \cup \operatorname{pcl}(B), \quad \operatorname{spcl}(A \cup B) \subseteq \operatorname{spcl}(A) \cup \operatorname{spcl}(B)$ $[12,13]$.

(4) $\operatorname{pint}(A \cap B) \subseteq \operatorname{pint}(A) \cap \operatorname{pint}(B), \operatorname{pint}(A \cup B) \supseteq \operatorname{pint}(A) \cup \operatorname{pint}(B)$ [14].

(5) $X /(\operatorname{int}(A))=\operatorname{cl}(X /(A)), \operatorname{int}(X / A)=X / \operatorname{cl}(A)$.

\section{$2 b^{*}$-Open sets}

Definition 2.1: let $(X, \tau)$ be topological space. Then a subset $A$ of Xis said to be

1. a $b^{*}$-Open set if $\mathrm{A} \subseteq \operatorname{cl}(\operatorname{int}(\operatorname{cl}(A))) \cup \operatorname{int}(\operatorname{cl}(A))$.

2. a $b^{*}$-closed set if $\mathrm{A} \supseteq \operatorname{int}(\operatorname{cl}(\operatorname{int}(A))) \cap \operatorname{cl}(\operatorname{inl}(A))$.

The family of all $b^{*}$-Open set (resp. $b^{*}$-closed set)subsets of a spase $(X, \tau)$ will be as always denoted by $b O^{*}(X)\left(\right.$ resp. $\left.b C^{*}(X)\right)$

Example 2.1: Let $X=\{a, b, c, d\}$ with topology $\tau=\{X, \phi,\{a\},\{a, b\},\{a, c, d\}\}$. Then the classes of $b^{*}$-open set and $b^{*}$-closed set

$b^{*} O(X)=\{X, \Phi,\{a\},\{a, b\},\{a, c\},\{a, d\},\{a, d\},\{a, b, c\},\{a, b, d\},\{a, c, d\}\}$, and

$b^{*} C(X)=\{X, \Phi,\{b\},\{c\},\{d\},\{c, d\},\{b, d\},\{b, c\},\{b, c, d\}\}$

Proposition 2.1: Let A be a sub set of a space $(X, \tau)$. Then (1) Every preopen (resp. Bc-open) set is $b^{*}$-open

Remark 2.1: The converse of the above propsition is not necessarily true as shown by the following example.

*Corresponding author: Sayed MEL, Professor, Department Mathematics, College of Science and Arts, Najran University, Saudi Abrabia, Tel:+966 17542 8888; E-mail: fa-hamad@hotmail.com

Received November 07, 2016; Accepted November 28, 2016; Published November 30, 2016

Citation: Sayed MEL, Mansour FHAL (2016) New Near Open Set In Topological Space. J Phys Math 7: 204. doi: 10.4172/2090-0902.1000204

Copyright: @ 2016 Sayed MEL, et al. This is an open-access article distributed under the terms of the Creative Commons Attribution License, which permits unrestricted use, distribution, and reproduction in any medium, provided the original author and source are credited. 
Example 2.2: Let $X=\{a, b, c\}$ with topology $\tau=\{X, \Phi\{a\},\{b\},\{a, b\}\}$. Then

(1) A subset $\{\mathrm{a}, \mathrm{c}\}$ of $\mathrm{X}$ is $b^{*}$-open but not preopen.

(2) A subset $\{\mathrm{a}\}$ of $\mathrm{X}$ is $b^{*}$-open but not Bc-open.

Remark 2.2: According to Definition (2.1) and Proposition (2.1), the following diagram holds for a subset $A$ of a space $X$ :

Lemma 2.1: Let $(X, \tau)$ be topological space. Then the following statements are hold

(1) The union of $b^{*}$-Open sets is $b^{*}$-open

(2) The intersection of $b^{*}$-closed sets is $b^{*}$-closed

Proof: (1) let $\left\{A_{i}, i \in I\right\}$ be a family of $b^{*}$-Opensets. Then $A_{i} \subseteq \operatorname{cl}\left(\operatorname{int}\left(\operatorname{cl}\left(A_{i}\right)\right)\right) \cup \operatorname{int}\left(\operatorname{cl}\left(A_{i}\right)\right)$, hence $U_{i} A_{i} \subseteq U_{i}\left(\operatorname{cl}\left(\operatorname{int}\left(\operatorname{cl}\left(A_{i}\right)\right)\right) \cup \operatorname{int}\left(\operatorname{cl}\left(A_{i}\right)\right)\right) \subset \operatorname{cl}\left(\operatorname{int}\left(\operatorname{cl}\left(U_{i} A_{i}\right)\right)\right) \cup \operatorname{int}\left(\operatorname{cl}\left(U_{i} A_{i}\right)\right)$, for all $i$. Thus $U_{i} A_{i}$ is $b^{*}$-Open

(2) let $\left\{A_{i}, i \in I\right\}$ be a family of $b^{*}-\operatorname{closed} \operatorname{sets}$. Then $A_{i} \supseteq \operatorname{int}\left(\operatorname{cl}\left(\operatorname{int}\left(A_{i}\right)\right)\right) \cap \operatorname{cl}\left(\operatorname{inl}\left(A_{i}\right)\right)$, hence $\bigcap_{i} A_{i} \supseteq \bigcap_{i}\left(\operatorname{int}\left(\operatorname{cl}\left(\operatorname{int}\left(A_{i}\right)\right)\right) \cap \operatorname{cl}\left(\operatorname{inl}\left(A_{i}\right)\right)\right) \supset \operatorname{int}\left(\operatorname{cl}\left(\operatorname{int}\left(\bigcap_{i} A_{i}\right)\right)\right) \cap \operatorname{cl}\left(\operatorname{inl}\left(\bigcap_{i} A_{i}\right)\right)$. for all $i \in I$. Thus $\bigcap A_{i}$ is $b^{*}$ closed

Remark 2.3: The intersection of any two $b^{*}$-open sets is not $b^{*}$-open. Let $X=\{a, b, c, d\}$,

$\tau=\{X, \Phi\{a\},\{c, d\},\{a, c, d\}\}$. Then $A=\{a, b\}$ and $B=\{b, c\}$ are $b^{*}$-open sets, but $A \cap B=\{b\}$ is not $b^{*}$-open.

Definition 2.2: Let $(X, \tau)$ be topological space. Then:

(1) The union of all $b^{*}$-open sets of $X$ contained in $A$ is called the $b^{*}$-interior of $A$ and is denoted by $b^{*}$-int $(A)$.

(2) The intersection of all $b^{*}$-closed sets of $X$ contained in $A$ is called the $b^{*}$-closure of $A$ and is denoted by $b^{*}-C l(A)$.

Example 2.3: Let $X=\{a, b, c, d\}$ with topology $\tau=\{X, \Phi\{a\},\{c\},\{a, c\}\}$. and $A=\{a, b\}, B=\{a, c\}$ are $b^{*}$ open then

$b^{*}-\operatorname{int}(A)=\{a, b\}, b^{*}-\operatorname{int}(B)=\{a, c\}$ and $b^{*}-c l(A)=\{a, b\}, b^{*}-c l(B)=X$

Theorem 2.1: Let $(X, \tau)$ be topological space and $A \subset X$, then the following statement are equivalent:

(1) $A$ is a $b^{*}$-open set,

(2) $A=\operatorname{spint}(A) \cup \operatorname{pint}(A)$

Proof: $(1) \rightarrow(2)$. Let $A$ be a $b^{*}$-open set. Then $A \subseteq \operatorname{cl}(\operatorname{int}(\operatorname{cl}(A))) \cup \operatorname{int}(\operatorname{cl}(A))$, hence by proposition (1.1).

$\operatorname{spint}(A) \cup \operatorname{pint}(A)=(A \cap \operatorname{cl}(\operatorname{int}(\operatorname{cl}(A))) \cup(A \cap \operatorname{int}(\operatorname{cl}(A)))=A \cap(\operatorname{cl}(\operatorname{int}(\operatorname{cl}(A))) \cup \operatorname{int}(\operatorname{cl}(A)))=A$

$(2) \rightarrow(1)$. Suppose that $A=\operatorname{spint}(A) \cup \operatorname{pint}(A)$. Than by proposition (1.1)

$A=\left(A \cap \operatorname{cl}(\operatorname{int}(\operatorname{cl}(A))) \cup(A \cap \operatorname{int}(\operatorname{cl}(A))) \subset \operatorname{cl}(\operatorname{int}(\operatorname{cl}(A))) \cup \operatorname{int}(\operatorname{cl}(A))\right.$. Therefore, $A$ is a $b^{*}$-open.

Theorem 2.2: Let $(X, \tau)$ be topological space and $A \subset X$, then the following statement are equivalent:

(1) $A$ is a $b^{*}$-closed set,

(2) $A=\operatorname{spcl}(A) \cap \operatorname{pcl}(A)$

Proof: (1) $\rightarrow(2)$ Let $A$ be a $b^{*}$-closed set. Then $A \supseteq \operatorname{int}(\operatorname{cl}(\operatorname{int}(A))) \cap \operatorname{cl}(\operatorname{int}(A))$, hence by proposition(1.1).

$\operatorname{spcl}(A) \cap \operatorname{pcl}(A)=(A \cup \operatorname{int}(\operatorname{cl}(\operatorname{int}(A))) \cap(A \cup \operatorname{cl}(\operatorname{int}(A)))=A \cup(\operatorname{int}(\operatorname{cl}(\operatorname{int}(A))) \cap \operatorname{cl}(\operatorname{int}(A)))=A$

$(2) \rightarrow(1)$. Suppose that $A=\operatorname{spcl}(A) \cap \operatorname{pcl}(A)$. Than by proposition (1.1)

$A=\left(A \cup \operatorname{int}(\operatorname{cl}(\operatorname{int}(A))) \cap(A \cup \operatorname{cl}(\operatorname{int}(A))) \supset \operatorname{int}(\operatorname{cl}(\operatorname{int}(A))) \cap \operatorname{cl}(\operatorname{int}(A))\right.$. Therefore, $A$ is a $b^{*}-\operatorname{closed}$.

Theorem 2.3: Let $A$ be a supset of a space $(X, \tau)$. Then

(1) $b^{*}-\operatorname{cl}(A)=\operatorname{spcl}(A) \cap \operatorname{pcl}(A)$,

(2) $b^{*}-\operatorname{int}(A)=\operatorname{spint}(A) \cup \operatorname{pint}(A)$.

Proof: (1) It is easy to see that $b^{*}-\operatorname{cl}(A) \subseteq \operatorname{spcl}(A) \cap \operatorname{pcl}(A)$. Also $\operatorname{spcl}(A) \cap p c l(A)=\left(A \cup \operatorname{int}(\operatorname{cl}(\operatorname{int}(A))) \cap(A \cup \operatorname{cl}(\operatorname{int}(A)))=A \cup(\operatorname{int}(\operatorname{cl}(\operatorname{int}(A))) \cap \operatorname{cl}(\operatorname{int}(A)))\right.$. But, $b^{*}-$ $c l(A)$ is $b^{*}$-closed, hence $x \quad A \cup(\operatorname{int}(\operatorname{cll}(\operatorname{int}(A))) \cap \operatorname{cl}(\operatorname{int}(A))) \subset A \cup b^{*}-\operatorname{cl}(A)=b^{*}-\operatorname{cl}(A)=b^{*}$ Thus $A \cup(\operatorname{int}(\operatorname{cl}(\operatorname{int}(A))) \cap \operatorname{cl}(\operatorname{int}(A))) \subset A \cup b^{*}-\operatorname{cl}(A)=b^{*}-\operatorname{cl}(A)=b^{*}$ there for, $\operatorname{spcl}(A) \cap \operatorname{pcl}(A) \subset b^{*}-\operatorname{cl}(A)$. So $b^{*}-\operatorname{int}(A) \subseteq \operatorname{spint}(A) \cup \operatorname{pint}(A)$.

(2) It is easy to see that $b^{*}-\operatorname{int}(A) \subseteq \operatorname{spint}(A) \cup \operatorname{pint}(A)$. Also $\operatorname{spint}(A) \cup \operatorname{pint}(A)=(A \cap \operatorname{cl}(\operatorname{int}(\operatorname{cl}(A))) \cup(A \cap \operatorname{int}(\operatorname{cl}(A)))=A \cap(\operatorname{cl}(\operatorname{int}(\operatorname{cl}(A))) \cup \operatorname{int}(\operatorname{cl}(A)))$. But, $b^{*}-\operatorname{int}(A)$ is $b^{*}-o p e n$, hence $b^{*}-\operatorname{int}(A) \subset \operatorname{cl}\left(\operatorname{int}\left(\operatorname{cl}\left(b^{*}-\operatorname{int}(A)\right)\right)\right) \cup \operatorname{int}\left(\operatorname{cl}\left(b^{*}-\operatorname{int}(A)\right)\right) \subset(\operatorname{cl}(\operatorname{int}(\operatorname{cl}(A))) \cup \operatorname{int}(\operatorname{cl}(A)))$. Thus $A \cap(\operatorname{cl}(\operatorname{int}(\operatorname{cl}(A))) \cup \operatorname{int}(c l(A))) \subset A \cap b^{*}-\operatorname{int}(A)=b^{*}-\operatorname{int}(A)$ there for $\operatorname{spint}(A) \cup \operatorname{pint}(A) \subset b^{*}-\operatorname{int}(A)$.

So $b-\operatorname{int}(A)=\operatorname{spint}(A) \cup \operatorname{pint}(A)$. 
Theorem 2.4: Let $A$ be a supset of a space $(X, \tau)$. Then

(1) $\mathrm{A}$ is a $b^{*}$-open set if and only if $A=b^{*}-\operatorname{int}(A)$

(2) $\mathrm{A}$ is a $b^{*}$-closed set if and only if $A=b^{*}-c l(A)$

Proof: (1) Let A be a $b^{*}$-open set. Then by theorem (2.1), $A=\operatorname{spint}(A) \cup \cup \operatorname{pint}(A)$ and by theorem (2.3), we have $A=b^{*}$-int $(A)$ Conversely, let $A=b^{*}-\operatorname{int}(A)$ Then by theorem(2.3), $A=\operatorname{spint}(A) \cup \operatorname{pint}(A)$ and by theorem (2.1), A is $b^{*}$-open $A=\operatorname{spint}(A) \cup \operatorname{pint}(A)$

(2) Let A be a $b^{*}$-closed set. Then by theorem (2.1), $A=\operatorname{spcl}(A) \cap p c l(A)$ and by theorem (2.3), we have $A=b^{*}-c l(A)$ Conversely, let $A=b^{*}-c l(A)$ Then by theorem (2.3), $A=\operatorname{spcl}(A) \cap \operatorname{pcl}(A)$ and by theorem (2.1), A is $b^{*}$-closed

Theorem 2.5: Let $A$ and $B$ be a subsets of a space $(X, \tau)$. Then the following are hold

(1) $b^{*}-\operatorname{cl}(X \backslash A)=X \backslash b^{*}-\operatorname{int}(A)$.

(2) $b^{*}-\operatorname{int}(X \backslash A)=X \backslash b^{*}-\operatorname{cl}(A)$.

(3) If $A \subseteq B$, then $x \in b^{*}-c l(A)$

(4) $x \in b^{*}-c l(A)$ if and only if there exists a $b^{*}$-open set $U$ and $x \in U$ such that $U \cap A \neq \phi$.

(5) $x \in b^{*}-\operatorname{int}(A)$ if and only if there exists a $b^{*}$-open set $G$ and $x \in G$ such that $x \in G \subseteq A$

(6) $b^{*}-\operatorname{cl}\left(b^{*}-\operatorname{cl}(A)\right)=b^{*}-c l(A)$ and $b^{*}-\operatorname{int}\left(b^{*}-\operatorname{int}(A)\right)=b^{*}-\operatorname{int}(A)$.

(7) $b^{*}-\operatorname{cl}(A) \cup b^{*}-\operatorname{cl}(B) \subseteq b^{*}-\operatorname{cl}(A \cup B)$ and $b^{*}-\operatorname{int}(A) \cup b^{*}-\operatorname{int}(B) \subseteq b^{*}-\operatorname{int}(A \cup B)$

(8) $b^{*}-\operatorname{int}(A \cap B) \subseteq b^{*}-\operatorname{int}(A) \cap b^{*}-\operatorname{int}(B), b^{*}-\operatorname{cl}(A \cap B) \subseteq b^{*}-\operatorname{cl}(A) \cap b^{*}-\operatorname{cl}(B)$

Proof: (1) Since $(X \backslash A) \subseteq X$, by theorem (2.4) $\quad b^{*}-\operatorname{cl}(X \backslash A)=\operatorname{spcl}(X \backslash A) \cap \operatorname{pcl}(X \backslash A) \quad$ and by proposition $\quad(1.1)$ $b^{*}-c l(X \backslash A)=(X \backslash \operatorname{spint}(A)) \cap(X \backslash \operatorname{pint}(A))=X \backslash(\operatorname{sint}(A) \cup \operatorname{pint}(A))$, hence by theorem $(2.4), b^{*}-c l(X \backslash A)=X \backslash b^{*}-\operatorname{int}(A)$

(2) Since $(X \backslash A) \subseteq X$, by theorem (2.4) $\quad b^{*}-\operatorname{int}(X \backslash A)=\operatorname{spint}(X \backslash A) \cup \operatorname{pint}(X \backslash A) \quad$ and $\quad$ by proposition $\quad(1.1)$ $b^{*}-\operatorname{int}(X \backslash A)=(X \backslash \operatorname{spcl}(A)) \cup(X \backslash \operatorname{pcl}(A))=X \backslash(\operatorname{spcl}(A) \cap \operatorname{pcl}(A))$, hence by theorem $(2.4), b^{*}-\operatorname{int}(X \backslash A)=X \backslash b^{*}-\operatorname{cl}(A)$

(3) Since, $b^{*}-\operatorname{cl}(A)=\operatorname{spcl}(A) \cap \operatorname{pcl}(A)$ and $A \subseteq B, b^{*}-\operatorname{cl}(A)=\operatorname{spcl}(A) \cap \operatorname{pcl}(A) \subseteq \operatorname{spcl}(B) \cap \operatorname{pcl}(B)=b^{*}-\operatorname{cl}(B)$

(4) Let $x \notin b^{*}$-cl $(A)$ then $x \notin \bigcap F$ where $\mathrm{F}$ is $b^{*}$-closed with $A \subset F$, so $x \notin X \backslash \cap F$ and $X \backslash \cap F$ is a $b^{*}$-open set containing $\mathrm{x}$ and hence $(X \backslash \bigcap F) \cap A \subseteq(X \backslash \bigcap F) \cap(\bigcap F)=\phi$. Converly, suppose that exists a $b^{*}$-open set containing $\mathrm{x}$ with $A \cap U=\phi$.

Then $A \subseteq X / U$ and $X / U$ is a $b^{*}$-closed. Hence $x \notin b^{*}-c l(A)$.

(5) Necessity. Let $x \in b^{*}$-int $(A)$. Then $x \in \cup\left\{G: G\right.$ is $b^{*}-$ open $\left.G \subseteq A\right\}$ and hence there exists $b^{*}$-open set $G$ such that $x \in G \subseteq A$ sufficiency. Let $G$ be a $b^{*}$-open set such that $x \in G \subseteq A$. Then $A=\cup\{G: x \in G\}$ which is the union of $b^{*}$-open set. There for, $x \notin b^{*}-c l(A)$.

(6) Since $b^{*}-\operatorname{cl}\left(b^{*}-\operatorname{cl}(A)\right)=\operatorname{spcl}\left(b^{*}-\operatorname{cl}(A)\right) \cap \operatorname{pcl}\left(b^{*}-\operatorname{cl}(A)\right)$. by theorem $(2.4)$.

$\operatorname{spcl}(\operatorname{spcl}(A) \cap \operatorname{pcl}(A)) \cap \operatorname{pcl}(\operatorname{spcl}(A) \cap \operatorname{pcl}(A)) \subseteq(\operatorname{spcl}(A) \cap \operatorname{spcl}(\operatorname{pcl}(A))) \cap \operatorname{pcl}(\operatorname{spcl}(A) \cap \operatorname{pcl}(A))=\operatorname{spcl}(A) \cap \operatorname{pcl}(A)=b^{*}-\operatorname{cl}(A)$ hence:

$b^{*}-\operatorname{cl}\left(b^{*}-\operatorname{cl}(A)\right) \subseteq b^{*}-\operatorname{cl}(A)$. But, $b^{*}-\operatorname{cl}(A) \subseteq b^{*}-\operatorname{cl}\left(b^{*}-\operatorname{cl}(A)\right)$, there for, $b^{*}-\operatorname{cl}\left(b^{*}-\operatorname{cl}(A)\right)=b^{*}-\operatorname{cl}(A)$

(7) Since $A \subseteq A \cup B$ and $B \subseteq A \cup B$ we have $b^{*}-c l(A) \subseteq b^{*}-c l(A \cup B)$ and $b^{*}-c l(B) \subseteq b^{*}-c l(A \cup B)$. There for $b^{*}-c l(A) \cup b^{*}-c l(B) \subseteq b^{*}-c l(A \cup B) A \subseteq(A \cup B)$ and and $B \subseteq(A \cup B)$ we have $b^{*}-\operatorname{int}(A) \subseteq b^{*}-\operatorname{int}(A \cup B)$ and $b^{*}-\operatorname{int}(B) \subseteq b^{*}-\operatorname{int}(A \cup B)$. There for $b^{*}-\operatorname{int}(A) \cup b^{*}-\operatorname{int}(B) \subseteq b^{*}-\operatorname{int}(A \cup B)$.

(8) Since $A \supseteq A \cap B$ and $B \supseteq A \cap B$ we have $b^{*}-c l(A) \supseteq b^{*}-c l(A \cap B)$ and $b^{*}-c l(B) \supseteq b^{*}-c l(A \cap B), b^{*}-c l(B) \supseteq b^{*}-c l\left((A \cap B)\right.$. There for for $b^{*}-c l(A) \cap$ $b^{*}-c l(B) b^{*}-c l(A \cap B)$ and $A(A \cap B)$ and $B \supseteq(A \cap B)$ we have $b^{*}-\operatorname{int}(A) \supseteq b^{*}-\operatorname{int}(A \cap B)$ and $b^{*}-\operatorname{int}(B) \supseteq b^{*}$-int $(A \cap B)$. There for $b^{*}-\operatorname{int}(A) \cap b^{*}-\operatorname{int}(B) \supseteq b^{*}$ $\operatorname{int}(A \cap B)$.

Remark 2.4: The inclusion relation in part (6),(7) of the above theorem cannot be replaced by equality as shown by the following example.

Example 2.4: Let $X=\{a, b, c, d\}$ with topology $\tau=\{X, \Phi\{a\},\{b\},\{c\}\{a, b\},\{a, c\}\{b, c\}\{b, d\}\{a, b, c\}\{a, b, d\}\{b, c, d\}\}$.

Then $(A \cup B)=\{a, b, d\}$

(1) If $\mathrm{A}=\{\mathrm{a}, \mathrm{b}\}, \mathrm{B}=\{\mathrm{d}\}$ and $(A \cup B)=\{a, b, d\}$, then $b^{*}-\operatorname{int}(A)=A b^{*}-\operatorname{int}(B)=\phi$ and $b^{*}-\operatorname{int}(A \cup B)=\{a, b, c\}$ So, $b^{*}-\operatorname{int}(A \cup B) \nsubseteq b^{*}-\operatorname{int}(A) \cup b^{*}-\operatorname{int}(B)$

(2) If $\mathrm{C}=\{\mathrm{b}\}, \mathrm{B}=\{\mathrm{d}\}$ and $(B \cap C)=\phi$, then $b^{*}-c l(C)=\{b, d\} b^{*}-c l(B)=B$ and $b^{*}-c l(B \cap C)=\phi$, there for, $b^{*}-c l(B) \cap b^{*}-c l(C) \nsubseteq b^{*}-c l(B \cap C)$

Example 2.5: Let $X=\{a, b, c, d\}$ with topology $\tau=\{X, \Phi\{a\},\{b, c\},\{a, b, c\}\}$ then

(1) If $\mathrm{A}=\{\mathrm{a}, \mathrm{b}\}, \mathrm{B}=\{\mathrm{a}, \mathrm{c}\}$ and $(A \cup B)=\{a, b, c\}$, then $b^{*}-c l(A)=A b^{*}-c l(B)=B$ and $b^{*}-c l(A \cup B)=X$ So, $b^{*}-c l(A \cup B) \nsubseteq b^{*}-c l(A) \cup b^{*}-c l(B)$

(2) If $\mathrm{C}=\{\mathrm{a}, \mathrm{d}\}, \mathrm{D}=\{\mathrm{b}, \mathrm{d}\}$ and $(B \cap C)=\{d\}$, then $b^{*}-\operatorname{int}(C)=C b^{*}-\operatorname{int}(D)=D$ and $b^{*}-\operatorname{int}(C \cap D)=\phi b^{*}-\operatorname{int}(C) \cup b^{*}-\operatorname{int}(D) b^{*}-\operatorname{int}(C \cap D)$

3 Some Topological Operations. 
Definition 3.1: Let $(X, \tau)$ be a space and $A \subset X$. Then the $b^{*}$-boundary of $A\left(\right.$ briefly, $\left.b^{*}-b(A)\right)$ is given by $\left.\left.b^{*}-b(A)\right)=b^{*}-c l(A)\right) \cap b^{*}-c l(X / A)$

Example 3.1: From Example (2.1) we have $A=\{a\} B=\{a, b\} C=\{a, b, d\}$ then $\left.\left.b^{*}-b(A)\right)=\{b, c, d\}, b^{*}-b(B)\right)=\{c, d\}$ and $\left.b^{*}-b(C)\right)=\{c\}$

Remark 3.1: For any subset $A$ of a space $(X, \tau)$ we have $b^{*}-b(A) \subseteq b(A)$ and $b^{*}-b(A) \subseteq p-b(A)$.

The inclusion of the above remark can be replaced as shows in the following example.

Example 3.2: From Example (2.3) and $A=\{a, b\}$ then $b^{*}-b(A)=\phi, p-b(A)=\{b, d\}$ we have $p-b(A) \nsubseteq b^{*}-b(A)$.

Theorem 3.1: If $A$ is a sub sets of a space $(X, \tau)$, then the following statement are hold:

(1) $\left.\left.b^{*}-b(A)\right)=b^{*}-b(X \backslash A)\right)$.

(2) $\left.\left.b^{*}-b(A)\right)=b^{*}-c l(A)\right) \backslash b^{*}-\operatorname{int}(A)$.

(3) $\left.b^{*}-b(A)\right) \cap b^{*}-\operatorname{int}(A)=\Phi$.

(4) $\left.b^{*}-b(A)\right) \cup b^{*}-\operatorname{int}(A)=b^{*}-\operatorname{cl}(A)$.

Proof: (1) Since $\left.\left.\left.\left.b^{*}-b(A)\right)=b^{*}-c l(A)\right) \cap b^{*}-\operatorname{cl}(X \backslash A)=b^{*}-b(X \backslash A)\right)=b^{*}-\operatorname{cl}(X \backslash A)\right) \cap b^{*}-\operatorname{cl}(A)$

(2)Since, $\left.\left.\left.b^{*}-b(A)\right)=b^{*}-\operatorname{cl}(A)\right) \cap b^{*}-\operatorname{cl}(X \backslash A)=b^{*}-\operatorname{cl}(A)\right) \cap\left(X \backslash b^{*}-\operatorname{intl}(A)\right)=\left(b^{*}-\operatorname{cl}(A) \cap X\right) \backslash\left(b^{*}-\operatorname{cl}(A) \cap X\right) \backslash\left(b^{*}-\operatorname{cl}(A) \cap b^{*}-\operatorname{intl}(A)\right)=b^{*}-\operatorname{cl}(A) \backslash b^{*}-\operatorname{int}(A)$.

(3) Also, by using (2) $\left.b^{*}-b(A)\right) \cap b^{*}-\operatorname{int}(A)=\left(b^{*}-c l(A) \backslash b^{*}-\operatorname{int}(A)\right) \cap b^{*}-\operatorname{int}(A)=\left(b^{*}-\operatorname{cl}(A) \cap b^{*}-\operatorname{int}(A)\right) \backslash b^{*}-\operatorname{int}(A)=b^{*}-\operatorname{int}(A) \backslash b^{*}-\operatorname{int}(A)=\Phi$.

(4) By using (3) $\left.b^{*}-b(A)\right) \cup b^{*}-\operatorname{int}(A)=\left(b^{*}-\operatorname{cl}(A) \backslash b^{*}-\operatorname{int}(A)\right) \cup b^{*}-\operatorname{int}(A)=b^{*}-\operatorname{cl}(A)$.

Theorem 3.2: If $A$ is a sub sets of a space $(X, \tau)$, then the following statement are holds:

(1) $A$ is a $b^{*}$-open set if and only if $A \cap b^{*}-b(A)=\Phi$

(2) $A$ is a $b^{*}$-closed set if and only if $b^{*}-b(A) \subset A$

(3) $A$ is a $b^{*}$-clopen set if and only if $b^{*}-b(A)=\Phi$.

Proof: (1) let A is a $b^{*}$-open set. Then $A=b^{*}-\operatorname{int}(A)$ hence by theorem (3.1)

$A \cap b^{*}-b(A)=b^{*}-\operatorname{int}(A) \cap b^{*}-b(A)=\Phi$ Conversely, let $A \cap b^{*}-b(A)=\Phi$ then by theorem (3.1),

$A \cap\left(b^{*}-\operatorname{cl}(A) \backslash b^{*}-\operatorname{int}(A)\right)=\left(A \cap\left(b^{*}-\operatorname{cl}(A)\right) \backslash\left(A \cap b^{*}-\operatorname{int}(A)\right)=A / b^{*}-\operatorname{int}(A)=\Phi\right.$. so, $A=b^{*}-\operatorname{int}(A)$ and hence $\mathrm{A}$ is $b^{*}-o p e n$.

(2) let $\mathrm{A}$ is a $b^{*}$-closed set. Then $A=b^{*}-\operatorname{cl}(A)$, by thearom (3.1), but $b^{*}-b(A)=\left(b^{*}-\operatorname{cl}(A) \backslash b^{*}-\operatorname{int}(A)\right)=A \backslash b^{*}-\operatorname{int}(A)$, then $b^{*}-b(A) \subset A$ Conversely let $b^{*}-b(A) \subset A$. Then by theorem(3.1), $\left.b^{*}-c l(A)=b^{*}-b(A)\right) \cup b^{*}-\operatorname{int}(A) \subset A \cup b^{*}-\operatorname{int}(A)=A$ thus $b^{*}-c l(A) \subset A$ and $A \subset b^{*}-c l(A)$ there for, $A=b^{*}-$ $\operatorname{cl}(A)$,

(3) let $A$ is a $b^{*}$-clopen set. Then $A=b^{*}-\operatorname{int}(A)$, and $A=b^{*}-\operatorname{cl}(A)$, hence by thearom (3.1), $b^{*}-b(A)=\left(b^{*}-\operatorname{cl}(A) \backslash b^{*}-\operatorname{int}(A)\right)=A \backslash A=\Phi$ Conversely, suppose that $b^{*}-b(A)=\Phi$. Then $b^{*}-b(A)=\left(b^{*}-\operatorname{cl}(A) \backslash b^{*}-\operatorname{int}(A)\right)=\Phi$, and hence, $A$ is a $b^{*}$-clopen set.

Definition 3.2: Let $(X, \tau)$ be a space and $A \subset X$.Then the $\operatorname{set} X \backslash\left(b^{*}-c l(A)\right)$ is called the $b^{*}$-exterior of $A$ and is denoted by $b^{*}$-ext $(A)$. Each point $p \in X$ is called an $b^{*}$-exterior point of $A$, if it is a $b^{*}$-interior point of $X \backslash A$.

Example 3.3: let $X=\{a, b, c, d\}$ with topology $\tau=\{X, \Phi\{a\},\{b\},\{a, b\},\{b, c, d\}\}$

If $A=\{a\} B=\{a, c\} C=\{b, c, d\}$ then we have

$\left.b^{*}-\operatorname{ext}(A)=\{b, c, d\}, b^{*}-\operatorname{ext}(B)\right)=\{b, d\}$ and $\left.b^{*}-b(C)\right)=\{a\}$

Remark 3.2: For any topology space $(X, \tau)$ and $A \subset X$, we have ext $(A) \subseteq p-\operatorname{ext}(A) \subseteq b^{*}-\operatorname{ext}(A)$

Proof: Since $b^{*}-c l(A) \subseteq \operatorname{cl}(A)$, then $X \backslash c l(A) \subseteq X \backslash b^{*}-\operatorname{cl}(A)$ and $\operatorname{int}\left(X \backslash A \subseteq b^{*}-\operatorname{int}(X \backslash A)\right.$ i.e $\operatorname{ext}(A) \subseteq b^{*}-\operatorname{ext}(A)$. Since $\operatorname{ext}(A) \subseteq p-\operatorname{ext}(A)$, then we have $p-\operatorname{ext}(A) \subseteq b^{*}-\operatorname{ext}(A)$. This implies that the relation hold.

Example 3.4: Let $X=\{a, b, c, d\} \quad$ with $\quad$ topology $\quad \tau=\{X, \Phi \quad\{c\},\{d\}\{c, d\},\{b, c\}\{b, c, d\}\} . \quad$ And $\quad A=\{b, d\}, \quad B=\{c\} \quad$ we $\quad h a v e$ $b^{*}-\operatorname{ext}(A)=\{a, c\}, p-\operatorname{ext}(A)=\{c\}, p-\operatorname{ext}(B)=\{b, d\}, \operatorname{ext}(B)=\{d\}$

Theorem 3.3: If $A$ and $B$ is two sub sets of aspace $(X, \tau)$, then the following statements are hold: ext $(A) \cup b^{*}$

(1) $b^{*}-\operatorname{ext}(A)=b^{*}-\operatorname{int}(A)$.

(2) $b^{*}-\operatorname{ext}(A)$ is $b^{*}$-open

(3) $b^{*}-\operatorname{ext}(A) \cup b^{*}-\operatorname{int}(A)=$.

(4) $b^{*}-\operatorname{ext}(A) \cap b^{*}-b(A)=$. 
(5) $b^{*}-\operatorname{ext}(A) \cup b^{*}-b^{*}-b(A)=b^{*} \quad c l(X \backslash A)$.

(6) $\left\{b^{*}-\operatorname{int}(A), b^{*}-b(A)\right.$ and $\left.b^{*}-\operatorname{ext}(A)\right\}$ from apartition of X.

(7) If $A \subseteq B$, then $b^{*}-\operatorname{ext}(B) \subseteq b^{*}-\operatorname{ext}(A)$

(8) $b^{*}-\operatorname{ext}(A \cup B) \subseteq b^{*}-\operatorname{ext}(A) \cup b^{*}-\operatorname{ext}(B)$.

(9) $b^{*}-\operatorname{ext}(A \cap B) \supseteq b^{*}-\operatorname{ext}(A) \cap b^{*}-\operatorname{ext}(B)$.

(10) $b^{*}-\operatorname{ext}(X)=\Phi$ and $b^{*}-\operatorname{ext}(\phi)=X$.

Proof: (1) by Definition (3.2) $b^{*}-\operatorname{ext}(A)=X \backslash b^{*}-c l(A)=b^{*}-\operatorname{int}(X \backslash A)$.

(2) From $(1) b^{*}-\operatorname{ext}(A)=b^{*}-\operatorname{int}(X \backslash A)$. Since $b^{*}-\operatorname{int}(A)$ is the union of all $b^{*}$-open sets of $X$ contained in $A$ thus $b^{*}-\operatorname{ext}(A)$ is $b^{*}$-open

(3) Since $b^{*}-\operatorname{ext}(A) \cap b^{*}-\operatorname{int}(A)=X \backslash b^{*}-\operatorname{cl}(A) \cap b^{*}-\operatorname{int}(A)=b^{*}-\operatorname{int}(X \backslash A) \cap b^{*}-\operatorname{int}(A)=\phi$

(4) By theorem (3.1), $b^{*}-\operatorname{ext}(A) \cap b^{*}-b(A)=b^{*}-\operatorname{int}(X \backslash A) \cap b^{*}-b(X \backslash A)=\Phi$.

(5) Also, by theorem (3.1)

$b^{*}-\operatorname{ext}(A) \cup b^{*}-b(A)=b^{*}-\operatorname{int}(X \backslash A) \cup b^{*}-b(X \backslash A)=b^{*}-c l(X \backslash A)$.

(6) From (3),(4) we have $b^{*}-\operatorname{ext}(A) \cap b^{*}-\operatorname{int}(A)=\Phi$ and $b^{*}-\operatorname{ext}(A) \cap b^{*}-b(A)=\Phi$. Then by theorem (3.1) then $\left.b^{*}-b(A)\right) \cap b^{*}-\operatorname{int}(A)=\Phi$. Now, we need to prove that $b^{*}-\operatorname{int}(A) \cup b^{*}-b(A) \cup b^{*}-\operatorname{ext}(A)=X$ hence from $(5) b^{*}-\operatorname{ext}(A) \cup b^{*}-b(A)=b^{*}-\operatorname{cl}(X \backslash A)$ then $b^{*}-\operatorname{int}(A) \cup b^{*}-c l(X \backslash A)=b^{*}-\operatorname{int}(A) \cup X \backslash b^{*}-\operatorname{int}(A)=X$.

(7) let $A \subseteq B$ then $\left(b^{*}-\operatorname{cl}(A)\right) \subseteq\left(b^{*}-\operatorname{cl}(B)\right)$ and hence

$X \backslash\left(b^{*}-c l(B)\right) \subseteq X \backslash\left(b^{*}-c l(A)\right)$. So $b^{*}-\operatorname{ext}(B) \subseteq b^{*}-\operatorname{ext}(A)$.

(8) $b^{*}-\operatorname{ext}(A \cup B)=X \backslash\left(b^{*}-c l(A \cup B)\right) \subseteq X \backslash\left(b^{*}-\operatorname{cl}(A) \cup\left(b^{*}-\operatorname{cl}(B)\right)==\left(X \backslash\left(b^{*}-\operatorname{cl}(A)\right)\right) \cap\left(X \backslash\left(b^{*}-c l(B)\right)\right)=b^{*}-\operatorname{ext}(A) \cap b^{*}-\operatorname{ext}(B) \subseteq b^{*}-\operatorname{ext}(A) \cup b^{*}-\operatorname{ext}(B)\right.$.

(9) $b^{*}-\operatorname{ext}(A \cap B)=X \backslash\left(b^{*}-c l(A \cap B)\right) \supset X \backslash\left(b^{*}-c l(A) \cap\left(b^{*}-c l(B)\right)=\left(X \backslash\left(b^{*}-c l(A)\right)\right) \cup\left(X \backslash\left(b^{*}-c l(B)\right)\right)=b^{*}-\operatorname{ext}(A) \cup b^{*}-\operatorname{ext}(B) \supseteq b^{*}-\operatorname{ext}(A) \cap b^{*}-\operatorname{ext}(B)\right.$.

(10) $b^{*}-\operatorname{ext}(X)=X \backslash\left(b^{*}-\operatorname{cl}(X)\right)=X \backslash X=\phi$ and $b^{*}-\operatorname{ext}(\phi)=X \backslash\left(b^{*}-\operatorname{cl}(\phi)\right)=X \backslash \phi=X$

Remark 3.3: The inclusion relation in part (5),(6) of the above theorem cannot by replaced by equality as is show by the following example.

Example 3.5: From Example (2.1) we have $A=\{b, c\}$ and $B=\{a, c\}$ then $b^{*}-\operatorname{ext}(A)=\{a, b\}, b^{*}-\operatorname{ext}(B)=\Phi$ but $b^{*}-\operatorname{ext}(A \cup B)=\Phi$. Therefor, $b^{*}-\operatorname{ext}(A) \cup b^{*}-\operatorname{ext}(B) \nsubseteq b^{*}-\operatorname{ext}(A \cup B)$. Also. $b^{*}-\operatorname{ext}(A \cap B)=\{a, b, d\}$, hence $b^{*}-\operatorname{ext}(A \cap B) \nsubseteq b^{*}-\operatorname{ext}(A) \cap b^{*}-\operatorname{ext}(B)$.

Definition 3.3: If $A$ is a subset of a space $(X, \tau)$, then a point $p X$ is called a $b^{*}$-limit point of a set $A \subset X$ if every $b^{*}$-open set $G \in X$ containing $p$ contains a point of $A$ other than $p$. The set of all $b^{*}$ - limit point of $A$ is called an $b^{*}$-derived set of $A$ and is denoted by $b^{*}-d(A)$

Example 3.6: let $X=\{a, b, c, d\}$ with topology $\tau=\{X, \Phi\{a\},\{c, d\},\{a, c, d\}\}$ and If $A=\{a, d\} B=\{a, c, d\}$ the $\left.b^{*}-d(A)\right)=\{\Phi\}$, and $\left.b^{*}-d(B)\right)=\{b\}$.

Theorem 3.4: If $A$ and $B$ is two sub sets of aspace $(X, \tau)$, then the following statements are hold:

(1) If $A \subset B$, then $b^{*}-d(A) \subset b^{*}-d(B)$.

(2) $A$ is a $b^{*}$-closed set if and only if it cantains each of its $b^{*}$-limit point.

(3) $b^{*}-\operatorname{cl}(A)=A \cup b^{*}-d(A)$.

(4) $b^{*}-d(A \cup B) \supset b^{*}-d(A) \cup b^{*}-d(B)$

(5) $b^{*}-d(A \cap B) \subset b^{*}-d(A) \cap b^{*}-d(B)$

Proof: (1) By definition (3.3), we have $p \in b^{*}-d(A)$ if and only if $G \cap(A \backslash\{P\}) \neq \phi$, for every $b^{*}$-open set G containing p. But $A \subset B$, then $G \cap(B \backslash$ $\{P\}) \neq \phi$, for every $b^{*}$-open set $\mathrm{G}$ containing $\mathrm{p}$. Hence, so $p \in b^{*}-d(B)$

There for $b^{*}-d(A) \subset b^{*}-d(B)$

(2) Let $\mathrm{A}$ be $b^{*}$-closed set and $p \notin A$ then $p \in(X / A)$ which is $b^{*}$-open, hence there exists $b^{*}$-open $(X \backslash A)$ such that

$(X / A) \cap A=\phi$ so $p \notin b^{*}-d(A)$, there for $b^{*}-d(A) \subset A$. Conversely, suppose that $b^{*}-d(A) \subset A$ and $p \notin A$. Then $p \notin b^{*}-d(A)$, hence there exists $b^{*}$-open set G containing p such that $G \cap A=\phi$ and hence

$X \backslash A=\bigcup_{p \in \mathbb{A}}\left\{G, G\right.$ is $b^{*}$ open there for $\mathrm{A}$ is $b^{*}$-closed

(3) Since, $b^{*}-d(A) \subset b^{*}-c l(A)$ and $A \subset b^{*}-c l(A) \quad b^{*}-d(A) \cup A \subset b^{*}-c l(A)$. 
Conversely, suppose that $p \notin b^{*}-d(A) \cup A$ Then $p \notin b^{*}-d(A), p \notin A$ and hence there exists $b^{*}$-open set $\mathrm{G}$ containing $\mathrm{p}$ such that $G \cap A=\phi$. Thus $p \notin$ $b^{*}-c l(A)$ which implies thet $b^{*}-\operatorname{cl}(A) \subset b^{*}-d(A) \cup A$, there for, $b^{*}-\operatorname{cl}(A)=b^{*}-d(A) \cup A$.

(5) Since $A \supseteq A \cap B$ and $B \supseteq A \cap B$ we have $b^{*}-d(A) \supseteq b^{*}-d(A \cap B)$ and $b^{*}-d(B) \supseteq b^{*}-d(A \cap B)$. There for $b^{*}-d(A) \cap b^{*}-d(B) \supseteq b^{*}-d(A \cap B)$.

Definition 3.4: Let $(X$,$) be a space and A \subseteq X$. Then the $b^{*}$-border of $A\left(\right.$ briefly, $\left.b^{*}-B d(A)\right)$ is given by $\left.b^{*}-B d(A)\right)=A \backslash b^{*}-\operatorname{int}(A)$.

Example 3.7: Let $X=\{a, b, c, d\}$ with topologies $\tau=\{X,\{d\},\{a, d\},\{c, d\},\{b, d\},\{b, c, d\},\{a, b, d\},\{a, c, d\}\}$. If $A=\{a, c\}, B=\{c, d\}$ and $C=\{b, c\}$, then $b^{*}-$ $B d(A)=\{a, c\}, b^{*}-B d(B)=\phi$ and $b^{*}-B d(c)=\{c\}$.

Theorem 3.5: For a subset $A$ of a space and $X$, the following statements are hold:

(1) $\left.A=b^{*}-\operatorname{int}(A) \cup b^{*}-B d(A)\right)$,

(2) $\left.b^{*}-\operatorname{int}(A) \cap b^{*}-B d(A)\right)=\phi$,

(3) $\left.\left.b^{*}-B d(X)\right)=b^{*}-B d(\phi)\right)=\phi$,

(4) $b^{*}-B d\left(b^{*}-\operatorname{int}(A)\right)=\phi$,

(5) $b^{*}-\operatorname{int}\left(b^{*}-B d(A)\right)=\phi$,

(6) $\left.b^{*}-B d\left(b^{*}-B d(A)\right)=b^{*}-B d(A)\right)$,

(7) $\left.b^{*}-B d(A)\right)=A \cap b^{*}-c l(X \backslash A)$,

(8) $\left.b^{*}-B d(A)\right)=b^{*}-d(X \backslash A)$,

Proof: $\left.(1) b^{*}-\operatorname{int}(A) \cup b^{*}-B d(A)\right)=b^{*}-\operatorname{int}(A) \cup\left(A \backslash b^{*}-\operatorname{int}(A)\right)=\left(b^{*}-\operatorname{int}(A) \cup A\right) \backslash\left(b^{*}-\operatorname{int}(A) \cup b^{*}-\right.$ $\operatorname{int}(A))=A \backslash b^{*}-\operatorname{int}(A)=A$,

(2) $\left.b^{*}-\operatorname{int}(A) \cap b^{*}-B d(A)\right)=b^{*}-\operatorname{int}(A) \cap\left(A \backslash b^{*}-\operatorname{int}(A)=\left(b^{*}-\operatorname{int}(A) \cap A\right) \backslash\left(b^{*}-\operatorname{int}(A) \cap b^{*}-\operatorname{int}(A)\right)\right.$ $=b^{*}-\operatorname{int}(A) \backslash b^{*}-\operatorname{int}(A)=\phi$

(3) $\left.b^{*}-B d(X)\right)-X \backslash b^{*}-\operatorname{int}(X)=X \backslash X=\phi$ and $\left.b^{*}-B d(\phi)\right)=\phi \backslash b^{*}-\operatorname{int}(\phi)=\phi \backslash \phi=\phi$.

(4) $b^{*}-B d\left(b^{*}-\operatorname{int}(A)\right)=b^{*}-\operatorname{int}(A) \backslash b^{*}-\operatorname{int}(A)=\phi$.

(5)Since, $b^{*}-\operatorname{int}\left(b^{*}-B d(A)\right)=b^{*}-\operatorname{int}\left(A \backslash b^{*}-\operatorname{int}(A)\right)=b^{*}-\operatorname{int}(A) \backslash b^{*}-\operatorname{int}\left(b^{*}-\operatorname{int}(A)\right)=b^{*}-\operatorname{int}(A) \backslash b^{*}-\operatorname{int}(A)=\phi$

(6) Since, $b^{*}-B d\left(b^{*}-B d(A)\right)=b^{*}-B d(A) \backslash b^{*}-\operatorname{int}\left(b^{*}-B d(A)\right)=b^{*}-B d(A) \backslash \phi=b^{*}-B d(A)$,

(7) Also, from Theorem (2.5), $\left.\left.b^{*}-B d(A)\right)=A \backslash b^{*}-\operatorname{int}(A)\right)=A \backslash\left(X \backslash b^{*}-\operatorname{cl}(A)\right)=A \cap b^{*}-\operatorname{cl}(X \backslash A)$.

(8) Further, from Theorem 2.3.1 $\left.\left.b^{*}-B d(A)\right)=A \backslash b^{*}-\operatorname{int}(A)\right)=A \backslash\left(A \backslash b^{*}-d(A)\right)=b^{*}-d(X \backslash A)$.

Theorem 3.6: For a subset $A$ of a space and $X$, the following statements are equivalent

(1) $A$ is $b^{*}$-open,

(2) $A=b^{*}-\operatorname{int}(A)$,

(3) $b^{*}-B d(A)=\phi$.

Proof: (1) $\rightarrow(2)$ Obvious from Theorem (2.4).

(2) $\rightarrow(3)$. Suppose that $A=b^{*}-\operatorname{int}(A)$. Then by Definition (3.4),

$b^{*}-B d(A)=b^{*}-\operatorname{int}(A) \backslash b^{*}-\operatorname{int}(A)=\phi$

$(3) \rightarrow(1)$. Let $b^{*}-B d(A)=\phi$. Then by Definition (3.4), $A \backslash b^{*}-\operatorname{int}(A)=\phi$ and hence $A=b^{*}-\operatorname{int}(A)$.

Definition 3.5: A subset $N$ of a space $(X, \tau)$ is called $a b^{*}$-neighbourhood (briefly, $b^{*}$-nbd.) of a point $p \in X$ if there exists a $b^{*}$-open set $W$ such that $p \in X \subseteq N$. The class of all $b^{*}$-nbds of $p X$ is called the $b^{*}$-neighbourhood system of $p$ and denoted by $b^{*}-N p$.

Example 3.8: Let $X=\{a, b, c, d\}$ with topology $\tau=\{X, \Phi,\{a\},\{c\},\{a, b\},\{a, c\},\{a, b, c\}\}$, then $b^{*}-N_{a}=\{a, c\}$.

Remark 3.4: For any topology spase $(X$,$) and for each x \in X$ we have $N_{x} \subseteq p-N_{x} \subseteq b^{*}-N_{x}$.

Example 3.9: From Example (2.2). We have $\{a, c\} \in b^{*}-N_{c}$ but it is not in $p-N_{c}$ and not in $N_{c}$.

Theorem 3.7: A subset $G$ of a space $X$ is $b^{*}$-open if and only if it is $b^{*}-n b d$, for every point $p \in G$.

Proof: Necessity. Let $G$ be an $b^{*}$-open set.Then $G$ is a $b^{*}$-nbd. for each $p \in G$.

Sufficiency. Let $G$ be a $b^{*}-n b d$, for each $p \in G$. Then there exists a $b^{*}$-open set $W$ containing $p$ such that $p \in W \subseteq G$, so $G=\cup\{p: p \in W\}$. Therefore, $G$ is $b^{*}$-open. 
Theorem 3.8: For a space $(X, \tau)$. If $b^{*}-N p$ is the $b^{*}-n b d$. systems of a point $p \in X$, then the following statements are hold:

(1) $b^{*}-N_{p}$ is not empty and $p$ belongs to each member of $b^{*}-N_{p}$

(2) Each superset of the members of $b^{*}-N_{p}$ belongs to $b^{*}-N_{p}$,

(3) Each member $N \in b^{*}-N p$ is a superset of the member $W \in b^{*}-N p$, where $W$ is $b^{*}-n b d$ of each point $p \in W$.

Proof: (1) Since $X$ is a $b^{*}$-open set containing $p$, then $X \in b^{*}-N p$. So, $b^{*}-N p \neq \phi$. Also, if $N b^{*}-N p$, then there exists a $b^{*}$-open set $G$ such that $p \in G \subseteq N$. Therefore, $p$ belongs to each member of $b^{*}-N p$.

(2) Let $M$ be a superset of $N \in b^{*}-N_{p}$, then there exists a $b^{*}$-open set $G$ such that $p \in G \subseteq N \subseteq M$ which implies $p \in G \subseteq M$ and hence, $M$ is a $b^{*}$ neighbourhood of $p$. Therefore, $M \in b^{*}-N_{p}$

(3) Let $N$ be a $b^{*}$-neighbourhood of $p \in X$, then there exists a $b^{*}$-open set $W$ such that $p \in W \subseteq N$. Then by Theorem 2.5.1, W is a $b^{*}$-neighbourhood of each of its points.

Definition 3.6: For a space $(X, \tau)$, a subset $A$ of $X$ is said to be $b^{*}$-dense in $X$ if and only if $b^{*}$-cl $(A)=X$ The family of all $b^{*}$-dense sets in $(X, \tau)$ will be denoted by $b^{*}-D(X, \tau)$

Example 3.10: Let $X=\{a, b, c\}$ with topology $\tau=\left\{X, \Phi,\{a, b\}\right.$ If, $A=\{a, b\}$, and $b^{*}-c l(A)=X$ than $b^{*}$-dense in $X$.

Remark 3.5: Every $b^{*}$-dense set in a space $(X, \tau)$ is dense in $(X, \tau)$ by the fact that $b^{*}-c l(A) \subseteq c l(A)$, while the converse may not be true.

Example 3.11: Let $X=\{a, b, c, d\}$ with topology $\tau=\{X, \varphi,\{a, c\},\{b, d\},\{a, c, d\}\}$. If $A=\{b, c, d\}$, then $\operatorname{cl}(A)=X$ but $b^{*}-C l(A)=\{b, c, d\}$ Therefore, $A$ is dense in $X$ but not $b^{*}$-dense in $X$.

Theorem 3.9: For a space $(X, \tau)$ and $E \subseteq X$, the following statements are equivalent:

(1) $E$ is $b^{*}$-dense in $X$

(2) If $F$ is an $b^{*}$-closed set in $X$ containing $E$, then, $F=X$

(3) $M-\operatorname{int}(X / E)=\phi$.

Proof: $(1) \rightarrow(2)$. Let $E$ be an $b^{*}$ - dense set of $X$. Then $b^{*}-C l(E)=X$. But $F$ is an $b^{*}$-closed set contains $E$, then $b^{*}-C l(E) \subseteq F$ and therefore $F=X$.

$(2) \rightarrow(3)$. Since $b^{*}-C l(E)$ is an $b^{*}$-closed set contains $E$, By (2) we have $b^{*}-C l(E)=X$. Hence $\phi=X \backslash b^{*} p-\operatorname{cl}(E)=b^{*}-\operatorname{int}(X \backslash E)$.

$(3) \rightarrow(1)$. Since $b^{*}-\operatorname{int}(X / E)=\phi$. Then $b^{*}-C l(E)=X$ Hence $E$ is $b^{*}$-dense in $X$.

Proposition 3.1: For a space $(X, \tau)$, if $E \in b^{*}-D(X, \tau)$, then the following statements are hold:

(1) $b^{*}-b(E)=b^{*}-\operatorname{cl}(X \backslash E)$,

(2) $b^{*}-\operatorname{ext}(E)=\phi$.

Proof: (1) From Definition (3.1), we have $b^{*}-b(E)=b^{*}-c l(E) \cap b^{*}-c l(X \backslash E)$ and since $E \in b^{*}-D(X, \tau)$, then $b^{*}-b(E)=b^{*}-c l(X \backslash E)$

(2) Also, by From Definition (3.2), $b^{*}-\operatorname{ext}(E)=X \backslash b^{*}-c l(E)$ but $E \in b^{*}-D(X, \tau)$, then $b^{*}-\operatorname{ext}(E)=\phi$.

Definition 3.7: For a space $(X, \tau), A \subseteq X$ is called:

(1) $b^{*}$ - nowhere dense if $\operatorname{int}(A) \subseteq b^{*}-\operatorname{int}\left(b^{*}-c l(A)\right)=\phi$

(2) $b^{*}$ - residual if $b^{*}-c l(X \backslash A)=X$ or $b^{*}-$ int $(A)=\phi$

$b^{*}$ - nowhere dense is $b^{*}$-iresidual from the fact that $b^{*}$-int $(A) \subseteq b^{*}$-int $\left(b^{*}-c l(A)\right)$ for every $A \subseteq X$

Example 3.12: Let $X=\{a, b, c\}$ with topology $\tau=\{X, \Phi,\{a\},\{a, b\}\}$ and $A=\{b\}$ than $b^{*}$-int $\left(b^{*}-c l(A)\right)=\phi$. and $b^{*}$-int $(A)=\phi$ so $A$ is $b^{*}-$ nowhere dense and $b^{*}$-residual.

Proposition 3.2: $A$ subset $A$ of a space $(X, \tau), A \subseteq X$ is $b^{*}$-nowhere dense of $X$ if $A \subseteq b^{*}-C l\left(X / b^{*}-c l(A)\right)$.

Proof: Let $A$ is $b^{*}$ - nowhere dense then $b^{*}-\operatorname{int}\left(b^{*}-c l(A)\right)=\phi$.

Hence $\left.X \backslash b^{*}-\operatorname{int}\left(b^{*}-\operatorname{cl}(A)\right)\right)=b^{*}-\operatorname{cl}\left(X \backslash b^{*}-\operatorname{cl}(A)\right)=b^{*}-\operatorname{cl}\left(b^{*}-\operatorname{int}(X \backslash A)\right)=X \supseteq A$

Theorem 3.10: The $b^{*}$-boundary of each $b^{*}$-open (resp. $b^{*}$-closed) set is $b^{*}$-nowhere dense.

Proof: Let $A \in b^{*} O(X)$ then

$$
\begin{aligned}
& b^{*}-\operatorname{int}\left(b^{*}-\operatorname{cl}\left(b^{*}-b(A)\right)\right)=b^{*}-\operatorname{int}\left(b^{*}-\operatorname{cl}\left(b^{*}-\operatorname{cl}(A) \cap b^{*}-\operatorname{cl}(X \backslash A)\right)=b^{*}-\right. \\
& \operatorname{int}\left(b^{*}-\operatorname{cl}\left(b^{*}-\operatorname{cl}\left(b^{*}-\operatorname{int}(A) \cap\left(X \backslash b^{*} \operatorname{int}(A)\right)\right)\right) \subseteq b^{*}-\operatorname{int}\left(b^{*}-\operatorname{cl}\left(b^{*}-\operatorname{int}(A) \cap\left(X \backslash b^{*}-\operatorname{int}(A)\right) \subseteq b^{*}-\operatorname{int}\left(b^{*}\right.\right.\right.\right. \\
& \left.-\operatorname{cl}\left(b^{*}-\operatorname{int}(A) \cap\left(X \backslash b^{*}-\operatorname{cl}\left(b^{*}-\operatorname{int}(A)\right)\right)\right)\right) \subseteq b^{*}-\operatorname{cl}\left(b^{*}-\operatorname{int}(A) \cap\left(X \backslash b^{*}-\operatorname{cl}\left(b^{*}-\operatorname{int}(A)\right)\right)\right)=\phi
\end{aligned}
$$

Also if $A \in b^{*} C(X)$ Then 


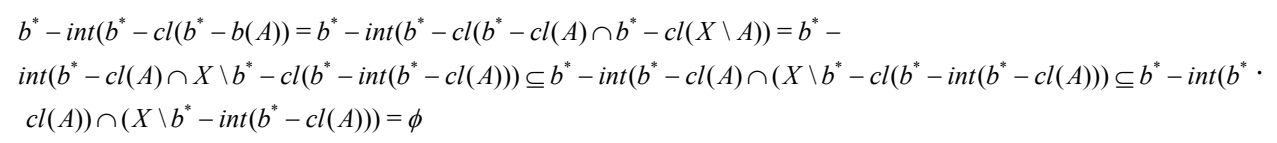

Proposition 3.3: For a space $(X, \tau), A \subseteq X$, then the sets $A \cap b^{*}-c l(X \backslash A)$ and $b^{*}-c l(A) \cap(X \backslash A)$ are $b^{*}$ - residual.

Proof: Since

$$
\begin{aligned}
& b^{*}-\operatorname{int}\left(A \cap b^{*}-\operatorname{cl}(X \backslash A)\right) \subseteq b^{*}-\operatorname{int}(A) \cap b^{*}-\operatorname{int}\left(b^{*}-\operatorname{cl}(X \backslash A)\right) \subseteq b^{*}-\operatorname{int}(A) \cap b^{*}- \\
& \operatorname{cl}(X \backslash A)=b^{*}-\operatorname{int}(A) \cap\left(X \backslash b^{*}-\operatorname{int}(A)\right)=\phi
\end{aligned}
$$

Then $A \cap b^{*}-c l(X \backslash A)$ is residual. Similarly

$b^{*}-\operatorname{int}\left(b^{*}-c l(A) \cap(X \backslash A)\right) \subseteq b^{*}-\operatorname{int}\left(b^{*}-\operatorname{cl}(A)\right) \cap b^{*}-\operatorname{int}(X \backslash A)=b^{*}-\operatorname{cl}(A) \cap\left(X \backslash b^{*}-\operatorname{cl}(A)\right)=\phi$, and hence $b^{*}-c(A) \cap(X \backslash A)$ is $b^{*}$ residual.

Theorem 3.11: The $b^{*}$-boundary of any set contains the union of two $b^{*}$-residual sets.

Proof: Let $(X, \tau)$ be a space and $A \subseteq X$. Then by Proposition (3.3), we have

$$
\begin{aligned}
& \left(A \cap b^{*}-\operatorname{cl}(X \backslash A)\right) \cup\left(b^{*}-c l(A) \cap(X \backslash A)\right)=\left(( A \cap b ^ { * } - \operatorname { c l } ( X \backslash A ) \cup b ^ { * } - \operatorname { c l } ( A ) ) \cap \left(\left(A \cup b^{*}-\right.\right.\right. \\
& \operatorname{cl}(X \backslash A)) \cup(X \backslash A)=\left(( A \cup b ^ { * } - \operatorname { c l } ( A ) ) \cap ( b ^ { * } - \operatorname { c l } ( X \backslash A ) \cup b ^ { * } - \operatorname { c l } ( A ) ) \cap \left(\left(A \cup ( X \backslash A ) \cap \left(b^{*}-\right.\right.\right.\right. \\
& \operatorname{cl}(X \backslash A) \cup(X \backslash A))=b^{*}-\operatorname{cl}(A) \cap\left(b^{*}-\operatorname{cl}(A) \cup b^{*}-\operatorname{cl}(X \backslash A)\right) \cap b^{*}-\operatorname{cl}(X \backslash A) \subseteq\left(b^{*}-\operatorname{cl}(A) \cap b^{*}-\right. \\
& c l(A \cup(X \backslash A))) \cap b^{*}-\operatorname{cl}(X \backslash A)=b^{*}-\operatorname{cl}(A) \cap b^{*}-\operatorname{cl}(X \backslash A)=b^{*}-b(A) .
\end{aligned}
$$

\section{References}

1. Bhattacharya P, Lahiri BK (1987) Semi generalized closed sets in topology. Indian J Math 29: 373-382.

2. Skowron A (1989) On topology information systems. Indian Bull Polish Acta Sci Math 36178: 87-90.

3. Pawlak Z (2012) Rough sets theatrical aspects of reasoning about data volume 9 of system theory knowledge Engineering and problems solving Kluwer Dordrecht.

4. Stone MH (1987) Applications of the theory of Boolean rings to general topology. Indian J Math 29: 373-382.

5. Levine N (1963) Semi open sets and semi continuous mappings in topological spaces. Amr Math Monthly 70: 36-41.

6. Najastad O (1965) On some classes of nearly open sets. Pacific J Math 15: 961-970.

7. Abd El-Monsef ME (1980) Studied on Some pre topological concepts. Ph.D. Thesis.

8. Mashhour AS, Abd El-Monsef ME, El-Deeb SN (1982) On pre continuous and 3. weak pre continuous mappings. Proc Math and phys Soc Egypt 53: 47-53.

9. Abd EL-Monsef ME, El-Deeb SN, Mahmoud RA(1983) $\beta$-open sets and $\beta$-continuous mappings. Bull Fac Sci Assiut Univ 12: 77-90.

10. Andrijevic D (1996) On b-open sets. Mat Vesnik 48: 59-64.

11. Ibrahim HZ Bc (2013) Open Sets in Topological Spaces. Advances in Pure Mathematics 3: 34-40.

12. Anitha M, Thangavelu P (2005) On Pre-Generalized Pre-Regular-Closed sets. Acta Ciencia Indica 31M: $1035-1040$.

13. Thangavelu P, Rao KC (2002) p-sets in topological spaces. Bulletin of Pure and Applied Sciences 21E: 341-355.

14. Navalgi GB (1998) pre-neighborhood. Matematics Edu. XXXII: 201-206.

15. Andrijevic D (1986) Semi preopen sets. Math. Vesnik 38: 24-32. 